\title{
A Method for Determining the Number of Dependent Constraints Using Transformation Parameters of Coordinate Systems
}

\author{
Korganbay Sholanov ${ }^{1, a^{*}}$ \\ ${ }^{1}$ Karaganda State Technical University, Mira blvd, 56, Karaganda city, 100027, \\ Republic of Kazakhstan \\ asholkor@jandex.kz
}

Keywords: Transformation parameters, Analysis, Degrees of freedom, Dependent constraints.

\begin{abstract}
This paper proposes a new method in which the number of dependent constraints is determined based on the transformation parameters of the coordinate systems associated with the links of a mechanism; the number of homonymous parameters that are equal to zero is subsequently used to determine the overall mobility (number of DOF) of the mechanism. This method can be used to solve problems involving the analysis and synthesis of the topological structure of any closed mechanism or parallel manipulator.
\end{abstract}

\section{Introduction}

The number of degrees of freedom (DOF) of a mechanism is determined by analysing its topological structure. Therefore, for instance, when solving a kinematics problem, the number of DOF determines the number of independent parameters in the position function. In the dynamics and control of mechanisms and manipulators, the number of independent dynamic equations and the number of actuators is equal to the number of DOF. This is why it is important to be able to accurately determine the number of DOF for single-loop and multi-loop mechanisms, including parallel manipulator mechanisms.

However, the analysis performed by Grigore Gogu [1] of studies published in the past 150 years indicates that to date, no theory or method for determining the number of DOF that is applicable to any arbitrary mechanism has been developed.

Therefore, the purpose of this paper is to obtain a universal and convenient method for determining the number of dependent constraints for subsequent DOF calculations concerning closed mechanisms and parallel manipulators. The objective is to use the coordinate transformation obtained from the mechanism's kinematics and its dynamical equation

\section{Choosing the Coordinate Systems for the Links}

According to kinematics [2], the position of a mechanism and the relative positions of its links can be determined using $A_{i}^{i-1}$ homogeneous transformation matrices. The elements of the $A_{i}^{i-1}$ matrices are the transformation parameters or trigonometric functions derived from them. The transformation parameters are the values of the linear or angular displacements that are required to merge the coordinate systems connected by joint-forming links. Fig. 1 shows the transformation parameters, $\delta_{l}$, $\theta_{l}, \alpha_{l}, \alpha_{i}, b_{i}$, and $\beta_{i}$, that are necessary to superpose the $O_{i-1} X_{i-1} Y_{i-1} Z_{i-1}$ and $O_{i} X_{i} Y_{i} Z_{i}$ coordinate systems. Here $A B=a_{i}$ - common perpendicular to the arbitrarily oriented axis $O_{i-1} Z_{i-1}$ and $O_{i} Z_{i}$. In this paper, the transformation parameters are used to define the positions of links relative to each other. It should be noted that this paper neither specifically defines the transformation parameters of the $A_{i}^{i-1}$ homogeneous transformation matrices nor solves matrix equations following the tradition of kinematics. Instead, the purpose of this paper is to demonstrate that the number of dependent constraints can be determined indirectly by analysing the transformation parameters. The solution to the problem begins with the recognition of the rules governing the $O_{i-1} X_{i-1} Y_{i-1} Z_{i-1}$ and $O_{i} X_{i} Y_{i} Z_{\mathrm{i}}$ 
coordinate systems associated with each pair of links, $i$ and $i-1$, between which $(i-1-i)$ joints are formed $(i=1, \ldots, n$, where $n$ is the total number of mobile links). A fixed link (frame) is denoted by the numeral zero (0). The main condition for selecting coordinate systems is that all of the kinematic variables describing the positions of the links and constant geometric quantities (the size of the links, the twist angles, etc.) must be included in the number of transformation parameters.

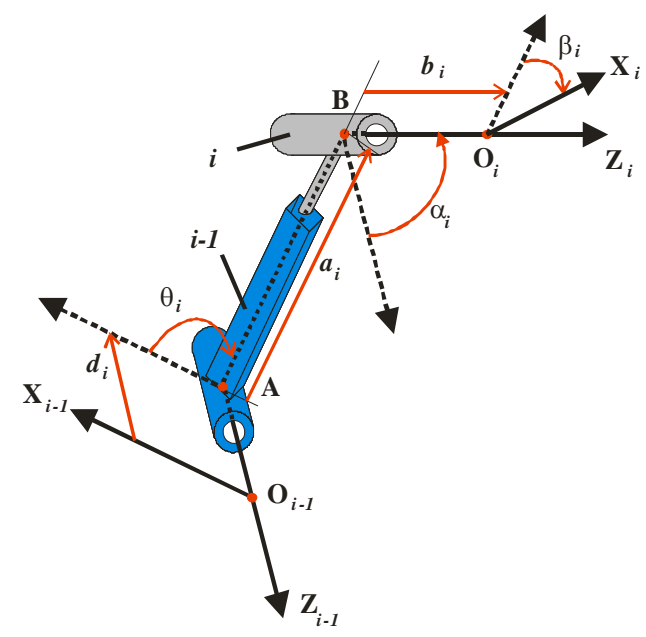

Fig. 1 The transformation parameters.

When choosing a coordinate system, the following are recommended:

- The $Z_{i-1}$ axes should be oriented along the axes of cylindrical, screw, prismatic, or revolute $(i-1)-i$ pairs or perpendicular to faces with slots for the fingers of two mobile spherical $(i-1)-i$ pairs.

- The origin of the $O_{i-1} X_{i-1} Y_{i-1} Z_{i-1}$ coordinate systems should be set at the centre of a spherical $(i-1)-i$ pair.

- The direction of the $X_{0}$ axis, which is associated with the support frame, must be chosen arbitrarily. The direction of the $X_{i}$ axis should be selected so that it is perpendicular to the plane that contains the $Z_{i-1}$ and $Z_{i}$ axes if these axes intersect; if these axes do not intersect, it should be perpendicular to the $Z_{i-1}$ and $Z_{i}$ axes. In a spherical kinematic pair with the finger, the $X_{i}$ axis should be directed along the axis of the finger.

Thus, the mutual locations of the links forming the $(i-1)-i$ joint is uniquely determined by six transformation parameters: three linear displacements, $d_{i}, a_{i}$, and $b_{i}$, along the generally non-coplanar $Z_{i-1}, A B$, and $Z_{i}$ axes and three rotation angles $\theta_{i}, \alpha_{i}$, and $\beta_{i}$ around the same axes. The basis vectors of the $Z_{i-1}, A B$, and $Z_{i}$ axes are the unit vectors $\vec{k}_{i-1}, \vec{e}_{i}$, and $\vec{k}_{i}$, respectively. The vector is used to provide vector designations for the transformation parameters, where the index $r=1, \ldots, 6$ denotes the number of the transformation parameter and the index $i=1, \ldots, n$ denotes the number of links and kinematical pairs of the mechanism, where $n$ is the total number of mobile links. Using this designation, the transformation parameter vectors can be written as follows:

$$
\vec{p}_{1 i}=d_{i} \vec{k}_{i-1}, \vec{p}_{2 i}=\theta_{i} \vec{k}_{i-1}, \vec{p}_{3 i}=a_{i} \vec{e}_{i}, \vec{p}_{4 i}=\alpha_{i} \vec{e}_{i}, \vec{p}_{5 i}=b_{i} \vec{k}_{i}, \vec{p}_{6 i}=\beta_{i} \vec{k}_{i}
$$

It should be noted, that in the cases where it is feasible, the Denavit Hartenberg method for selecting and transforming a coordinate system can be used. As it is known, in this case the four transformation parameters are used.

\section{An analysis of the Transformation Parameters}

The transformation parameters, $d_{i}, \theta_{v}, a_{i}, \alpha_{i}, b_{i}$, and $\beta_{i}$, may be variable, constant, or equal to zero. Particular note should be taken of the parameters that are equal to zero because they characterize peculiarities of the relative locations of the $O_{i-1} X_{i-1} Y_{i-1} Z_{i-1}$ and $O_{i} X_{i} Y_{i} Z_{i}$ coordinate systems. Therefore, the zero-valued parameters characterize peculiarities of the configuration of joints. Table 1 describes 
these peculiarities of the locations of the coordinate system in which the transformation parameters equal to zero .

Table 1. The parameters are zero and the corresponding of the peculiarities of the relative positions of the coordinate systems.

\begin{tabular}{|c|l|}
\hline $\begin{array}{c}\text { Transformation } \\
\text { parameter } \\
\text { equal to zero }\end{array}$ & $\begin{array}{l}\text { Peculiarities of the relative positions of the associated coordinate systems } \\
O_{i-1} X_{i-1} Y_{i-1} Z_{i-1} \text { and } O_{i} X_{i} Y_{i} Z_{i}\end{array}$ \\
\hline$d_{i}$ & $\begin{array}{l}\text { The origin of the } O_{i-1} X_{i-1} Y_{i-1} Z_{i-1} \text { coordinate system is located at point } A \text {, namely, } \\
\text { the intersection of the } O_{i-1} Z_{i-1} \text { axis with the mutual perpendicular } A B\end{array}$ \\
\hline$\theta_{i}$ & The $O_{i-1} X_{i-1}$ axis is oriented parallel to line $A B$ or perpendicular to the $Z_{i}$ axis \\
\hline$a_{i}$ & The $O_{i-1} Z_{i-1}$ and $O_{i} Z_{i}$ axes intersect \\
\hline$\alpha_{i}$ & The $O_{i-1} Z_{i-1}$ and $O_{i} Z_{i}$ axes are parallel \\
\hline$b_{i}$ & $\begin{array}{l}\text { The origin of the } O_{i} X_{i} Y_{i} Z_{i} \text { coordinate system is located at point } B, \text { namely, the } \\
\text { intersection of the } O_{i} Z_{i} \text { axis with the mutual perpendicular } A B\end{array}$ \\
\hline$\beta_{i}$ & The $O_{i} X_{i}$ axis is parallel to line $A B$ \\
\hline
\end{tabular}

\section{A Determination of the Number of Dependent Constraints and DOF}

Let us consider a mechanical system with holonomic stationary constraints that is composed of $n$ members $(i=1, \ldots, n)$. All of the forces acting on each link $i$, including inertia, are represented by 3 force components $F_{i}^{l}$ and 3 pairs of forces with moments $M_{i}^{l}(l=1,2,3)$ directed along the vectors $\vec{k}_{i-1}$, $\vec{e}_{i}$, and $\vec{k}_{i}(1)$. Then, all of the forces and moments can be represented using generalized force vectors.

$$
\vec{Q}_{1 i}=F_{i}^{1} \vec{k}_{i-1} ; \quad \vec{Q}_{2 i}=M_{i}^{1} \vec{k}_{i-1} ; \quad \vec{Q}_{3 i}=F_{i}^{2} \vec{e}_{i} ; \quad \vec{Q}_{4 i}=M_{i}^{2} \vec{e}_{i} ; \quad \vec{Q}_{5 i}=F_{i}^{3} \vec{k}_{i} ; \quad \vec{Q}_{6 i}=M_{i}^{3} \vec{k}_{i} .
$$

The general dynamical equation for the given system at a virtual displacement is

$$
\sum_{r=1}^{6} \delta \overline{\bar{p}}_{r i} \overline{\bar{Q}}_{r i}=0(i=1, \ldots, n)
$$

where $\bar{Q}_{r i}=\left(\begin{array}{llllll}\vec{Q}_{1 i} & \vec{Q}_{2 i} & \vec{Q}_{3 i} & \vec{Q}_{4 i} & \vec{Q}_{5 i} & \vec{Q}_{6 i}\end{array}\right)$ is a column vector whose elements are the generalized force vectors $\bar{Q}_{r i}(2)$ and $\delta \overline{\bar{p}}_{r i}=\left(\begin{array}{llllll}\delta \vec{p}_{1 i} & \delta \vec{p}_{2 i} & \delta \vec{p}_{3 i} & \delta \vec{p}_{4 i} & \delta \vec{p}_{5 i} & \delta \vec{p}_{6 i}\end{array}\right)^{\top}$ is a row vector whose elements are the possible displacement vectors of the transformation parameters given in (1).

The matrix equation given in (3) is equivalent to a linear system of equations of the form

$$
\delta d_{i} F_{i}^{1}+\delta \theta_{i} M_{i}^{1}+\delta a_{i} F_{i}^{2}+\delta \alpha_{i} M_{i}^{2}+\delta b_{i} F_{i}^{3}+\delta \beta_{i} M_{i}^{3}=0,(i=1, \ldots, n) .
$$

In this equation, it is understood that the forces and moments are variables and that the possible changes in the transformation parameters $\left(\begin{array}{llllll}\delta d_{i} & \delta \theta_{i} & \delta a_{i} & \delta \alpha_{i} & \delta b_{i} & \delta \beta_{i}\end{array}\right)$ are the coefficients of a system of Eq. (4) that describes a linear space of no more than 6 dimensions because the column rank of the $R$ matrix is limited to $6-\mathrm{R} \leq 6$.

If all of the links are rigidly connected by means 6 constraints imposed on each joint, then all of the equalities in Eq. (4) are satisfied, and the equation system has a rank of 6. From this, it follows that the system of equations given in Eq. (4) governs a constraint space and that the rank of the $R$ matrix, which defines the dimensionality of this space, corresponds to the number of independent constraints.

Changing the dimension of the space, i.e., the number of independent constraints, is possible when the transformation parameters are dependent or zero. Cases in which the transformation parameters are equal to zero due to the mutual orientation of the coordinate axes are discussed before. The possible dependency of the transformation parameters is discussed here. Suppose the $\mathrm{O}_{\mathrm{i}-1} \mathrm{Z}_{\mathrm{i}-1}$ and $\mathrm{O}_{\mathrm{i}} \mathrm{Z}_{\mathrm{i}}$ axes are parallel, then, the transformation parameters $d_{i}, b_{i}$ and $\theta_{l}, \beta_{l}$ characterize the linear and 
angular displacement in the same direction. Therefore, the expressions in Eq. (4) are similar and as a result, the number of similar terms is reduced to four. In this case, the duplicate terms should be set to zero $\left(b_{i}=\beta_{i}=0\right)$, which is equivalent to reducing the number of summands in Eq. (4). Conversion is also possible when there are dependent parameters. For example, transforming some pairs of parameters is equivalent to moving the system along an axis twice. Then, Eq. (3) is reduced by one summand because the expressions for the generalized forces of motion along the same axis are similar. In this case, the dependent parameters are both repeated, and one of them is zero. In this case, the system's coefficients are equal to zero when the corresponding transformation parameters are equal to zero. In some cases, for example, in the overconstrained linkages there are repetitive kinematic chains, which allow to derive additional equations for further processing. These equations reduce the number of independent Eq. (4), that is, every equation which is new to the system of four (4) equations, reduces the size of the constraint space. In order to define the conditions for determining the number of independent constraints in a closed kinematic chain, we will summarize in the four (4) equations the basic effects of similar forces and reaction force pairs (a vertical summation will be performed). Thus, the following equation is derived:

$$
\sum_{k=1}^{n} F_{k}^{1} \delta d_{k}+\sum_{k=1}^{n} M_{k}^{1} \delta \theta_{k}+\sum_{k=1}^{n} F_{k}^{2} \delta a_{k}+\sum_{k=1}^{n} M_{k}^{2} \delta \alpha_{k}+\sum_{k=1}^{n} F_{k}^{3} \delta b_{k}+\sum_{k=1}^{n} M_{k}^{3} \delta \beta_{k}=0
$$

To find out which of the summands in an equation becomes zero, let's review any given standalone summand, for example as follows:

$$
\sum_{k=1}^{n} F_{k}^{1} \delta d_{k}=F_{1}^{1} \delta d_{1}+F_{2}^{1} \delta d_{2}+\ldots+F_{n-1}^{1} \delta d_{n-1}+F_{n}^{1} \delta d_{n}=0
$$

In this example, the reaction forces cannot equal zero based on constraints of the given linkage. Transformation parameters in each linkage are independent values. Hence the following conclusion; this equation can only be executed on condition that all homonymous transformation parameters $\left(\mathrm{d}_{\mathrm{k}}\right.$ in this case) equal zero. Thus, if a number of $-S<6$ zero value homonymous transformation parameters is present in all of the four (4) system equations, then the rank of the coefficient matrix of the four (4) equations, and consequentially the dimensions of space reduce by the value of $S$. In this case, the maximum dimensions of the space described by the system (4) will equal $R=6-S$. Coefficients that are equal to zero correspond to parameters that are equal to zero, and it follows that the quantity $S$ is the smallest number of zero homonymous parameters present in each of the equations in the system. For example, if for some mechanism, in the system of six equations of the form given in Eq. (4), 3 of the parameters in the $1^{\text {st }}$ equation, 4 in the $2^{\text {nd }}, 4$ in the $3^{\text {rd }}, 3$ in the $4^{\text {th }}, 3$ in the $5^{\text {th }}$, and 2 in the $6^{\text {th }}$ are zero, then $S=2$ because no fewer than 2 parameters are equal to zero in any of the system's equations. In this instance, the dimensionality of the space is $R=6-S=4$. Therefore, the number of independent parameters defining constraints is 4 , and $S=2$ is the number of dependent constraints. It follows that the number of dependent constraints is equal to the smallest number of zero homonymous parameters present any of the equations of system (4). An analysis of the system's coefficient matrix (4) allows the number of dependent constraints to be determined. From a practical point of view, determining $\mathrm{S}$ for each closed loop mechanism or manipulator is sufficient to create a table (matrix) of the transformation parameters. Then, using the table, the smallest number of zero homonymous parameters in any row is determined. This determines the number of dependent constraints, $\mathrm{S}$.

For a one-loop mechanism, the number of DOF is

$W=M-6+S$.

The number of DOF of a multi-loop mechanism can be calculated using the following relation:

$$
W=M-6 k+\sum_{i=1}^{k} S_{1}
$$


where $k$ is the number of independent loops and $S_{i}$ is the number of dependent constraints on the $i$-th loop. A dependency similar to the one given in $(5,6)$ has been reported in reference [3].

\section{The Steps in Analysing the Structure of a Mechanism Using the Zero Transformation Parameters}

The sequence of steps in the analysis of a mechanism is as follows:

1. Assign numbers to the mobile links. The frame should be labelled 0 .

2. Define the number of independent loops.

3. Choose a coordinate system connected with the links in accordance with the recommendations given in Part 1.

4. Determine the transformation parameters and make a table of the transformation parameters in each loop by merging the coordinate systems in sequence (Part 2). Exclude the dependent parameters. Then, determine which row has the fewest parameters equal to zero. This minimum number of zero parameters is equal to the number of dependent constraints on the loop under analysis.

5. Use formula (5) or (6) to determine the number of DOF of the mechanism.

An analysis of the structures of mechanisms for which there is a divergence between the actual and calculated (using traditional methods) numbers of DOF is presented below.

\section{Samples Analyses of Mechanisms' Structures}

Let us consider a four-bar mechanism (Fig. 2) that is called the spherical mechanism, in which the axes of revolute pairs intersect at a point $\mathrm{O}$.

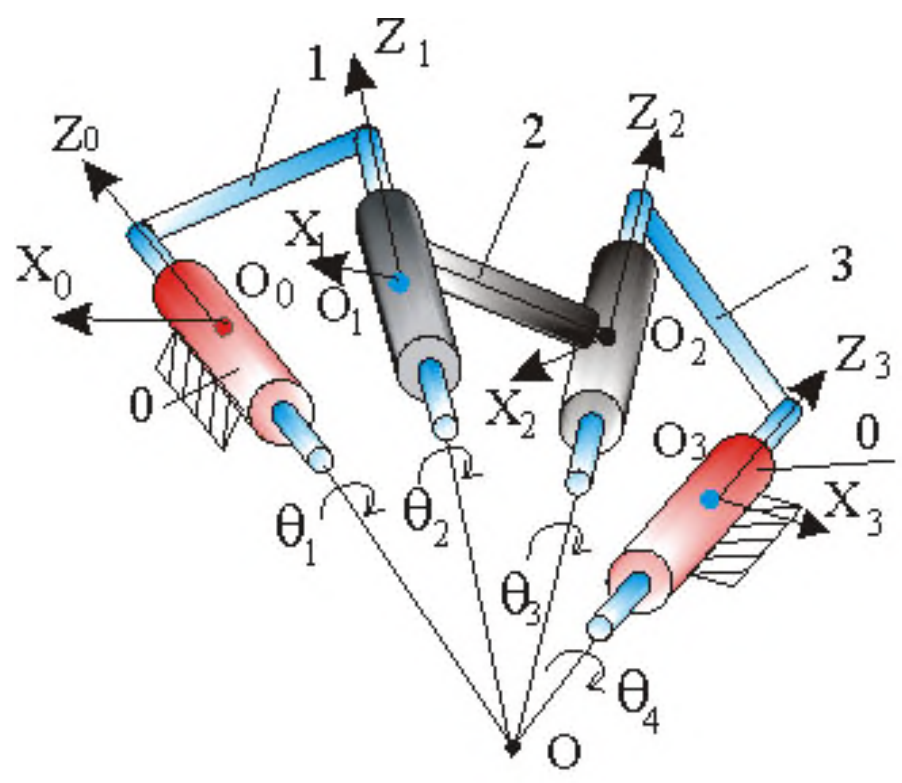

Fig. 2 The spherical mechanism.

In Fig. 2, the links are denoted 0-3 and the $Z_{i}$ axes associated with links that are oriented along the axes of revolute kinematic pairs are selected. The direction of the $X_{0}$ axis is chosen perpendicular to the plane $Z_{0} O Z_{3}$. The other $X_{\mathrm{I}}$ axes are perpendicular to the plane containing the $Z_{i-1}$ and $Z_{i}$ axes. To combine the coordinate systems $O_{0} X_{0} Z_{0}$ and $O_{1} X_{1} Z_{1}$, the following steps are taken. The system $O_{0} X_{0} Z_{0}$ is shifted along the $O_{0} Z_{0}$ axis by a distance $d_{1}=O_{0} O$. The $O X_{0}$ axis is rotated through an angle $\theta_{1}$ to align it with the $O_{1} X_{1}$ axis. The $O_{1} X_{1}$ axis is not shifted; therefore, $a_{1}=0$. From this position, the system is rotated through an angle $\angle \alpha_{1}=\angle O_{0} O O_{1}$. The system is shifted a distance $b_{1}=O O_{1}$ along the $O Z_{1}$. There is no need to rotate the $O_{1} Z_{1}$ axis; therefore, $\beta_{l}=0$. Similar actions are performed to identify the transformation parameters of the other kinematic pairs. As a result of this transformation, 
the dependent parameters, namely $b_{1}=d_{2}, b_{2}=d_{3}$, and $b_{3}=d_{4}, b_{4}=d_{1}$ are found. In accordance with the conclusions obtained in Part 4 , we set $b_{1}=b_{2}=b_{3}=b_{4}=0$. The transformation parameters for the kinematic pairs obtained after eliminating the dependent parameters are summarized in Table 2 .

Table 2. The transformation parameters for the spherical mechanism.

\begin{tabular}{|c|c|c|c|c|c|c|}
\hline \multirow{2}{*}{$\begin{array}{c}\text { Joint formed by the } \\
\text { specified links }\end{array}$} & \multicolumn{7}{|c|}{ Transformation parameters } \\
\cline { 2 - 7 } & $d_{i}$ & $\theta_{i}$ & $a_{i}$ & $\alpha_{i}$ & $b_{i}$ & $\beta_{i}$ \\
\hline $0-1$ & $d_{1}=O_{0} O$ & $\theta_{1}$ & 0 & $\alpha_{1}$ & 0 & 0 \\
\hline $1-2$ & $d_{2}=O O_{1}$ & $\theta_{2}$ & 0 & $\alpha_{2}$ & 0 & 0 \\
\hline $2-3$ & $d_{3}=O O_{2}$ & $\theta_{3}$ & 0 & $\alpha_{3}$ & 0 & 0 \\
\hline $3-0$ & $d_{4}=O O_{3}$ & $\theta_{4}$ & 0 & $\alpha_{4}$ & 0 & 0 \\
\hline
\end{tabular}

From an analysis of Table 2 , it is found that $M=4$, i.e., $\theta_{i}=\operatorname{Var}$, and $S=3$; therefore, according to formula (5), the number of DOF of this mechanism is $W=1$.

\section{Conclusions}

This paper proposes a method of kinematical analysis based on the use of the zero transformation parameters of coordinate systems to determine the number of dependent constraints, given the topological structure of a closed-loop mechanism or manipulator. It is proven that the number of homonymous transformation parameters that are zero depends on the rank of the coefficient matrix of the system of linear equations derived from the general dynamical equations. It is also found that the number of dependent constraints is equal to the smallest number of zero homonymous parameters (coefficients equal to zero) in any equation of the system. Once all of the dependent constraints have been found, the number of DOF of a single-loop or multi-loop closed mechanism can be determined based on the known dependencies.

\section{References}

[1] G. Grigore, Mobility of mechanisms: a critical review. Mech. Mach. Theory, 40(9) (2005) 1068-1087.

[2] P. N. Sheth, J.J. Jr. Uicker, A generalized symbolic notation for mechanisms, J. Eng. Ind. Trans. ASME, Series B, 93(1) (1971) 96-105.

[3] R. Voinea and M. Atanasiu, Contribution to the study of the structure of kinematic chanies. Bull. Ynst. Politehn. Bucuresti, t. XXII, Fasc. 1, 1960. 